\section{Syndrome inflammatoire de reconstitution immune}

> Le syndrome de reconstitution immune regroupe l'ensemble des manifestations inflammatoires survenant lors de la reconstitution d'une réponse immune excessive et insuffisamment régulée vis-à-vis d'antigènes infectieux ou non infectieux, chez un individu au décours d'une phase d'immunodépression. Ce syndrome est observé dans des contextes d'immunodépression/reconstitution très divers: reconstitution immune obtenue grâce aux antirétroviraux au cours de l'infection par le VIH, diminution d'un traitement immunosuppresseur au décours d'une transplantation, sortie d'aplasie après chimiothérapie, ou encore période du post-partum. La reconnaissance d'une entité unique commune à toutes ces situations pourra aider à en déterminer la physiopathologie qui reste encore incertaine. Cette compréhension est en effet un prérequis pour le développement de thérapies, en particulier préventives. <

\section{L'émergence d'un syndrome unique}

La reconstitution immune est le passage d'un état d'immunodépression acquis et réversible - lié à une pathologie (infection VIH, tuberculose, etc.) ou induit par une thérapeutique (chimiothérapie anticancéreuse, traitement immunosuppresseur) - à un état d'immunité fonctionnelle que provoque un traitement anti-infectieux ou l'arrêt d'un traitement immunosuppresseur. Cette reconstitution immune, bénéfique pour éradiquer un agent infectieux, peut cependant être délétère et entraîner des manifestations pathologiques que l'on désigne par le terme «syndrome inflammatoire de reconstitution immune» (ou IRIS, I'acronyme le plus utilisé) [1-3]. L'IRIS est attribué à une reconstitution immune excessive et/ou insuffisamment régulée et qui s'exerce vis-à-vis $d$ 'antigènes infectieux ou non infectieux après la modification du statut immunitaire d'un individu [1-3]. Les premières descriptions (avant que cette entité soit identifiée) remontent à l'utilisation de traitements antimy-

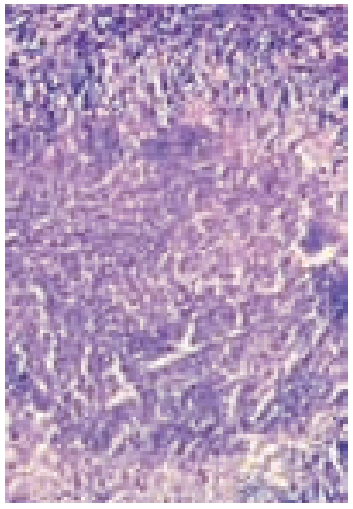

Service de médecine interne 1, Groupe hospitalier Pitié-Salpêtrière, 47-83, boulevard de l'Hôpital, 75013 Paris, France. guillaume.breton@ psl.aphp.fr

cobactériens pour combattre la tuberculose et la lèpre: des cas d'aggravation paradoxale (tuberculose) ou des réactions de réversion ${ }^{1}$ (lèpre) avaient été observées; le traitement, efficace sur le plan microbiologique, entraînait des manifestations pathologiques sous forme de fièvre, adénopathies, épanchements pleuraux, tuberculome intracrânien, chez des patients atteints de tuberculose et fièvre, adénopathie, aggravation de lésions cutanées ou nerveuses, uvéite, néphropathie chez les patients atteints de lèpre. Les mycobactéries sont en effet responsables d'une immunodépression et leur éradication par un traitement efficace restaure l'immunité avec la formation de lésions granulomateuses. L'identification de ce syndrome est contemporaine de l'apparition du Sida. Le nombre important d'infections et la reconstitution rapide de l'immunité que permettent les antirétroviraux (ART) ont en effet entrainé une augmentation considérable du nombre des IRIS [1-3]. Plus récemment, des manifestations pathologiques inflammatoires ont été observées dans plusieurs situations cliniques: après transplantation au sortir de la phase profonde d'immunodépression $[4,5]$, chez des patients cancéreux traités par chimiothérapie et dont la neutropénie était corrigée par l'administration de facteurs de croissance [6], après l'arrêt d'un traitement immunosuppresseur dans des maladies de système [7] et même, plus physiologiquement, au décours d'un accouchement [8]. On propose aujourd'hui de regrouper

${ }^{1}$ Réaction de réversion : exacerbation des lésions préexistantes avec parfois névrites aiguës sévères. 

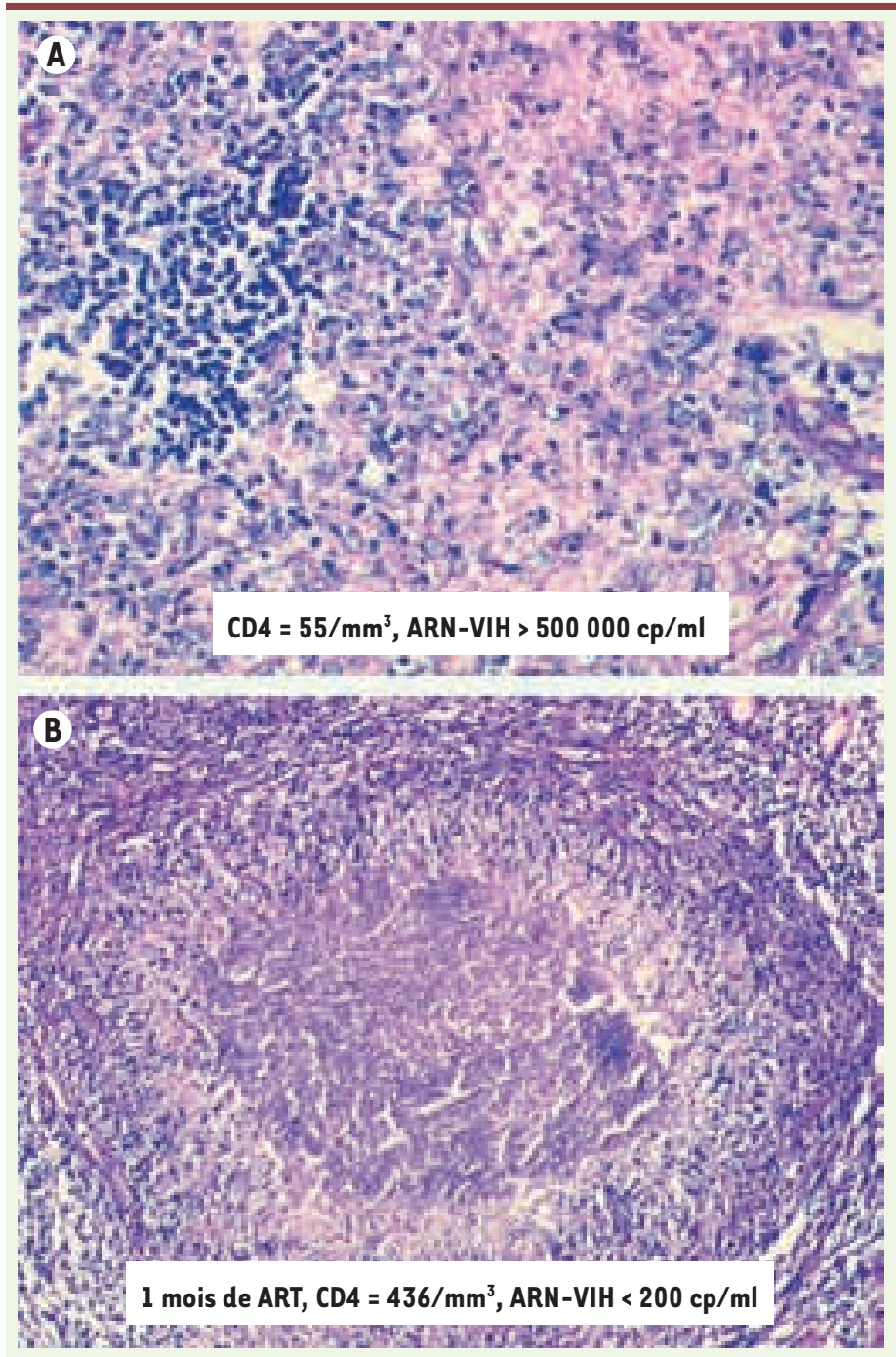

l'ensemble des manifestations sous le concept unique de syndrome inflammatoire de reconstitution immune. Les manifestations autoimmunes associées aux effets immunomodulateurs de l'interféron alpha sont sans doute assez similaires mais elles ne seront pas traitées ici car il ne s'agit pas de reconstitution immune au sens propre.

\section{Critères diagnostiques et classification des IRIS}

Plusieurs définitions et classifications ont été proposées pour les IRIS associés aux infections VIH [1-3, 9] (Tableau I). Un travail collaboratif international a récemment établi et validé des critères diagnostiques, utilisables en situation de ressources médicales limitées, pour les IRIS liés à la tuberculose chez les patients infectés par le VIH [10, 11]. II n'y a pas, à ce jour, de définition univoque d'un IRIS : cela s'explique par la multiplicité des formes cliniques, des agents infectieux impliqués et des causes d'immunodépression/reconstitution sous-jacente. Pour prendre en compte tous ces éléments, les critères diagnostiques doivent rester souples et tenir compte de nombreux critères d'exclusion qui ne peuvent parfois être reconnus que rétrospectivement. Le
Figure 1. Aspect anatomopathologique de la reconstitution immune fonctionelle lors d'un IRIS lié à Histoplasma capsulatum chez un patient infecté par le VIH après ART. A. Biopsie ganglionnaire lors du diagnostic d'histoplasmose montrant une architecture ganglionnaire détruite et un granulome histiocytaire riche en Histoplasma capsulatum (non montré) (Giemsa $x$ 100). B. Biopsie mésentérique réalisée lors d'une occlusion digestive fébrile en rapport avec un IRIS survenant 1 mois après le début du traitement antifongique alors que I'ARN du VIH est indétectable et que le taux des T CD4 est remonté à $436 / \mathrm{mm}^{3}$. L'histologie montre un granulome avec des cellules géantes contenant de rares levures (non montré) dont la culture restera négative (Giemsa x 100) [15].

diagnostic peut être évoqué devant l'association des critères suivants: (1) l'apparition de manifestations pathologiques marquées par des signes inflammatoires dans un contexte de modification de la fonctionnalité de la réponse immune. (2) Des manifestations qui restent inexpliquées: elles ne correspondent ni à un effet indésirable des traitements, ni à une infection nouvellement acquise, ni à un échec du traitement d'une infection préalablement identifiée (résistance, inobservance, etc.), ni à l'évolution attendue d'une infection ou d'une pathologie auto-immune/inflammatoire préalablement authentifiée.

De façon schématique, on peut distinguer trois formes différentes d'IRIS [9] :

- une forme d'IRIS paradoxal: le traitement d'une infection permet l'amélioration initiale des symptômes qui s'aggravent secondairement après la reconstitution immune. Les manifestations sont liées à la réponse immune vis-à-vis de fragments antigéniques de l'agent pathogène devenu inactif microbiologiquement;

- une forme d'IRIS infectieux: la reconstitution d'une réponse immune dirigée contre un agent infectieux quiescent mais viable peut démasquer une infection jusqu'alors asymptomatique ;

- une forme d'IRIS auto-immun qui regroupe les pathologies auto-immunes ou inflammatoires qui peuvent être déclenchées ou aggravées par la modification de la réponse immune.

\section{Hypothèses physiopathologiques de I'IRIS : le yin et le yang}

\section{Une réponse lymphocytaire Thl explosive}

dans les IRIS liés aux mycobactéries

et aux infections fongiques

Au cours de la tuberculose - qu'elle survienne ou non dans le contexte d'une infection par le VIH - des infec- 


\begin{tabular}{|c|c|c|}
\hline & Agent infectieux, non infectieux & Manifestations cliniques \\
\hline \multicolumn{3}{|c|}{ Infection à Mycobactérie après traitement antimycobactérien } \\
\hline \multirow[t]{2}{*}{ Mycobactéries } & M. tuberculosis & Fièvre, lymphadénite, lésion pulmonaire \\
\hline & M. leprae & Réaction de réversion \\
\hline \multicolumn{3}{|c|}{ Infection par le VIH après initiation du traitement antirétroviral } \\
\hline \multirow[t]{3}{*}{ Mycobactéries } & M. tuberculosis & Fièvre, lymphadénite, lésion pulmonaire \\
\hline & M. avium complex & Fièvre, lymphadénite \\
\hline & M. leprae & Réaction de réversion \\
\hline \multirow[t]{4}{*}{ Mycose } & C. neoformans & Méningite aseptique, lymphadénite \\
\hline & Histoplasma capsulatum & Lymphadénite \\
\hline & Pneumocystis jirovecii & Détresse respiratoire \\
\hline & Aspergillus fumigatus & Pneumopathie nécrosante \\
\hline \multirow[t]{8}{*}{ Virus } & VZV & Récurrence de zona \\
\hline & HSV & Ulcération chronique \\
\hline & CMV & Uvéite, pneumopathie \\
\hline & HHV8 & Sarcome de Kaposi extensif \\
\hline & $J C$ virus & Aggravation de LEMP \\
\hline & Hépatite $C$ et $B$ & Elévation des transaminases, séroconversion \\
\hline & HPV & Récurrence condylome \\
\hline & VIH & Encéphalomyélite, vascularite cérébrale \\
\hline Parasites & Leishmania infantum, major & Uvéite, dermatite post-kala-azar \\
\hline Maladies & Sarcoïdose & \\
\hline \multirow[t]{2}{*}{ inflammatoires } & Mie de Basedow & Apparition ou poussée évolutive \\
\hline & Syndrome de Guillain-Barré & \\
\hline \multicolumn{3}{|c|}{ Greffe d'organe après diminution des immuno-suppresseurs } \\
\hline Mycose & C. neoformans & Pneumopathie, méningite, adénopathie, fièvre \\
\hline \multirow[t]{2}{*}{ Virus } & CMV & Uvéite \\
\hline & BK virus & Cystite hémorragique \\
\hline \multicolumn{3}{|c|}{ Chimiothérapie aplasiante (cancer, hémopathie) en sortie d'aplasie } \\
\hline Bactéries & Staphylococcus aureus & Abcès, pyomyosite \\
\hline \multirow[t]{2}{*}{ Mycoses } & Aspergillus fumigatus & Aggravation pneumopathie \\
\hline & Candida species & Candidose hépatosplénique \\
\hline Pathologie inflammatoire & Psoriasis & Apparition \\
\hline \multicolumn{3}{|l|}{ Grossesse (fin $3^{\mathrm{e}}$ trimestre et post-partum) } \\
\hline \multirow[t]{2}{*}{ Mycobactérie } & M tuberculosis & Tuberculose extrapulmonaire \\
\hline & M leprae & Réaction de reversion \\
\hline \multirow[t]{2}{*}{ Mycoses } & C. neoformans & Méningite, pneumopathie \\
\hline & Coccidioides immitis & Infection disséminée \\
\hline \multirow[t]{3}{*}{ Virus } & Hépatite C, B & Elévation transaminase \\
\hline & HSV & Endométrite \\
\hline & HPV & Récurence condylome \\
\hline Pathologie inflammatoire/auto-immune & $\begin{array}{l}\text { Polyarthrite rhumatoïde, } \\
\text { sarcoïdose, } \\
\text { thyroïdite, } \\
\text { sclérose en plaques }\end{array}$ & Apparition ou poussée évolutive \\
\hline \multicolumn{3}{|l|}{ Arrêt anti-TNF } \\
\hline Bactéries & M. tuberculosis & Fièvre, lymphadénite, lésion pulmonaire \\
\hline
\end{tabular}

Tableau I. Principaux agents infectieux et non infectieux à l'origine de syndromes de reconstitution immunitaire liés à différentes étiologies d'immunodépression/reconstitution (adapté de [1]]). Notes: M, Mycobactérium; C, Cryptococcus ; CMV, cytomégalovirus; VZV, virus varicelle zona ; HSV, herpes simplex virus; HHV8, herpès virus du groupe 8 ; HPV, papilloma virus. LEMP : leucoencéphalopathie multifocale progressive. 
tions fongiques liées au VIH ou chez les patients greffés, et de la sarcoïdose, les IRIS semblent liés à la réapparition d'une hypersensibilité retardée $[3,9]$. Plusieurs éléments sont en effet évocateurs d'une reconstitution de la fonctionnalité des macrophages dans le cadre d'une réponse cellulaire spécifique de type Thl: la repositivation de l'intradermoréaction à la tuberculine observée au cours des IRIS liés à la tuberculose [12], la mise en évidence de granulomes épithélioïdes et surtout gigantocellulaires, parfois accompagnés de nécrose et de très rares agents infectieux. Ces granulomes se voient dans les IRIS liés à une tuberculose [13], à une cryptococcose [14] (patients infectés par le VIH ou greffés) [5], à une histoplasmose [15] (patients VIH), aux candidoses hépatospléniques (patients traités par chimiothérapie en sortie d'aplasie) [16] (Figure 1). Ces constatations sont confirmées par la forte réponse des lymphocytes T CD4 mémoires de type Thl visà-vis de M. tuberculosis, M. avium ou Cryptococcus neoformans, qui est observée au cours de I'IRIS chez les patients VIH [17, 18]. Cette polarisation Thl peut, par ailleurs, favoriser les rejets de greffe observés lors des IRIS [19].

L'importance de cette réponse Thl est corroborée par le fait que dans les différentes situations d'immunodépression/reconstitution à I'origine d'IRIS, comme l'infection VIH, les traitements immunosuppresseurs et la grossesse, le statut immunitaire évolue d'un profil anti-inflammatoire Th2 vers un statut pro-inflammatoire Thl après le début du traitement antirétroviral, la diminution des immunosuppresseurs, la sortie d'aplasie ou l'accouchement, pour garder les exemples cités ci-dessus.

\section{Un orage cytokinique}

Lors de I'IRIS, on observe des manifestations cliniques inflammatoires avec une fièvre souvent importante et des marqueurs biologiques de l'inflammation (protéine C réactive) très élevés. II s'y ajoute une forte production de l'ensemble des cytokines pro-inflammatoires, conséquence probable de l'absence de régulation de la réponse inflammatoire [17].

\section{Une insuffisance de contrôle de la réponse Thl}

L'activité des corticoïdes est démontrée dans I'IRIS lié aux mycobactéries et aux mycoses : elle diminue le taux des cytokines Thl, augmente celui des cytokines Th2 et stimule les lymphocytes T régulateurs, ce qui permet de contrôler cette réponse excessive. Cette action des corticoïdes pourrait traduire l'insuffisance des mécanismes de contrôle de la réponse pro-inflammatoire. Une des hypothèses est, en effet, que I'IRIS soit lié à un déséquilibre entre une réponse pro-inflammatoire bénéfique pour l'éradication d'un agent infectieux et une insuffisance de rétrocontrôle de cette réponse pro-inflammatoire possiblement relayée par les $T$ régulateurs dont la fonctionnalité semble altérée [20]. Le rôle de l'immunité innée, et en particulier des lymphocytes $\mathrm{T} \gamma \delta[21,41]$, des cellules natural killer (NK) et des macrophages, pourrait être important. À ce jour, on ne sait pas si la réponse Thl excessive que l'on observe au moment des symptômes cliniques de I'IRIS est secondaire ou non à une dérégulation de l'immunité innée qui serait l'élément déterminant initial.

\section{La piste des lymphocytes Th17}

La découverte récente de la voie de régulation Th17 [42] pourrait aussi avoir sa part de responsabilité dans la genèse de I'IRIS en association à ou indépendamment de la réponse Thl. La réponse Th17 est proinflammatoire, comme la réponse Thl, mais elle est en plus responsable de l'induction et du maintien de l'activation des polynucléaires neutrophiles. Cette dernière pourrait expliquer la suppuration fréquemment trouvée dans les IRIS associés aux infections mycobactériennes [3]. La spécification des lymphocytes vers la voie Th17 est régulée par I'IL(interleukine)-6 qui est très présente à la phase aiguë d'un état infectieux. On pourrait y voir une explication, lors des IRIS liés aux mycobactéries, au lien décrit entre la survenue d'IRIS, la dissémination de l'infection [9] et le polymorphisme de I'IL-6 [22].

\section{Les mystères des IRIS associés aux infections virales} Si d'authentiques IRIS ont été décrits lors d'infections virales (herpès virus, JC virus, virus des hépatites), ils sont rares et leur fréquence est surévaluée. L'aggravation, chez des patients mis sous ART, d'une leucoencéphalite multifocale progressive liée au virus JC traduit-elle un IRIS ou plutôt l'évolution naturelle de la maladie? En l'absence de critères radiologiques (prise de contraste, effet de masse) ou histologiques évocateurs (infiltration par des lymphocytes T CD8 périvasculaires), il est actuellement impossible de distinguer ces deux hypothèses. Or, dans les nombreux cas publiés d'IRIS associés au virus JC ou à d'autres infections virales, ces critères diagnostiques ne sont pas clairement définis. Parmi les manifestations liées aux virus survenant après l'introduction des ART, certaines sont au contraire des manifestations d'absence de reconstitution immune ou d'amplification paradoxale liée à une augmentation de la réplication virale. Cette dernière pourrait être la conséquence d'une prolifération virale stimulée par les cytokines pro-inflammatoires de type Thl normalement présentes lors de la reconstitution immune non pathologique (par exemple herpèsvirus humain 8 ou HHV8), mais aussi d'une compétition entre les virus VIH et VHC (virus de l'hépatite C) qui partagent des cibles cellulaires similaires, la clairance du VIH permettant d'augmenter les cibles pour le VHC et sa réplication.

Pour les cas confirmés d'IRIS survenant au cours des infections virales (herpès virus, JC virus, virus des hépatites), le mécanisme semble lié à une réponse T CD8 cytotoxique excessive comme en témoigne l'incidence accrue de zona après le début du traitement ART, liée au nombre de T CD8 circulants, l'identification d'infiltrats périvasculaires constitués de lymphocytes CD8 activés 
au cours d'IRIS liés au virus JC responsable de leucoencéphalopathies multifocales progressives fatales [23], et la forte prédominance de CD8 lors d'une uvéite à cytomégalovirus (CMV) chez un patient greffé [24]. La physiopathologie de ces IRIS reste incertaine et controversée et pourrait, comme précédemment, traduire la défaillance de mécanismes régulateurs des CD8 activés.

\section{La clé de I'IRIS, les polymorphismes?}

La question centrale de l'origine exacte de I'IRIS reste ouverte : pourquoi certains individus développent un IRIS alors que d'autres, qui partagent avec eux tous les facteurs de risques connus d'IRIS, restent indemnes? L'hypothèse avancée est celle de polymorphismes des génes codant des cytokines ou leurs récepteurs; c'est probablement le cas pour I'IL-6 dans les IRIS liés au VIH, de l'IL-4 pour ceux qui surviennent dans le contexte de candidoses hépatospléniques, de la lèpre et même de la grossesse (I'IL-12 serait impliquée dans ce dernier cas) $[8,16,22]$. Au cours des réactions de réversion de la lèpre, le rôle du polymorphisme d'un récepteur Toll-like (TLR-2) impliqué dans la présentation de l'antigène par la cellule dendritique, une étape décisive dans l'orientation de la balance Th1/Th2, a été récemment montré et pourrait être un des éléments clés de la genèse de
I'IRIS [25]. Cependant, la fréquence très élevée de survenue d'un IRIS, proche de $50 \%$ dans certaines situations, plaide en faveur de mécanismes multifactoriels s'ajoutant à un, ou des, polymorphismes : il peut s'agir de l'agent infectieux lui même, de l'environnement, de paramètres nutritionnels (carence en vitamine $D$ ), d'une immunomodulation liée aux hormones dans le contexte d'une grossesse, de l'hétérogénéité de la cinétique de reconstitution immune selon les types cellulaires, du cumul d'infections chez les patients très immunodéprimés à l'origine d'une hyperactivation immune, d'une tolérance immune favorisée par des infections digestives récidivantes d'origine bactérienne (lipopolysaccharide).

\section{Facteurs de risque de survenue du syndrome de reconstitution immunitaire}

\section{Facteurs de risque de survenue d'un IRIS}

Certains ont été identifiés.

- La profondeur du déficit immunitaire. Un taux de lymphocytes T CD4 inférieur à 50-100/mm3 [26-28] dans le contexte d'infections VIH compliquées ou non de tuberculose, une lymphopénie globale dans celui de tuberculoses hors du contexte d'une infection VIH [29], ou encore la sévérité (taux de polynucléaires neutrophiles inférieur à 100/ $\mathrm{mm}^{3}$ ) et la durée de la neutropénie dans les IRIS associés aux cryptococoses chez les patients greffés, aux candidoses hépatospléniques et aux aspergilloses pulmonaires en sortie d'aplasie $[5,6,16,30]$.

\section{Critères majeurs}

Présentation atypique d'une pathologie opportuniste ou tumorale chez des patients répondant au traitement antirétroviral

- maladie localisée

- réaction inflammatoire exagérée

- réponse inflammatoire atypique dans les tissus

- progression de l'atteinte d'un organe ou augmentation de taille de lésions préexistantes après une amélioration initiale sous traitement spécifique avant le début du traitement antirétroviral et après exclusion d'une toxicité médicamenteuse ou d'un autre diagnostic Diminution de la charge virale VIH $>1 \log _{10}$ copies/ml

\section{Critères mineurs}

- Augmentation du taux des lymphocytes T CD4 après traitement antirétroviral

- augmentation de la réponse immunitaire spécifique

- résolution spontanée sans traitement spécifique anti-infectieux ou antitumoral avec poursuite du traitement antirétroviral

\section{Classification}

1. Pathologie de reconstitution immunitaire infectieuse

- précoce (<3 mois après l'introduction du traitement antirétroviral) liée à une réponse immune vis-à-vis d'un agent infectieux opportuniste actif mais quiescent

- tardive (> 3 mois après l'introduction du traitement antirétroviral) liée à une réponse immunitaire vis-à-vis d'un antigène d'un agent infectieux non viable

2. Sarcoïdose de reconstitution immunitaire

3. Pathologie auto-immune de reconstitution immunitaire

Tableau II. Critères diagnostiques et classification du syndrome de reconstitution immunitaire. Le diagnostic d'IRIS nécessite 2 critères majeurs ou 1 critère majeur et 2 critères mineurs (adapté de [1]). 
- La dissémination de l'infection initiale, qu'il s'agisse d'une infection opportuniste (cryptocoque, CMV) ou de la tuberculose [9, 13, 14], ou de la localisation extrapulmonaire d'une tuberculose dans les IRIS liés à la tuberculose hors VIH [29].

- Le début précoce d'un traitement antirétroviral, moins de 4 à 6 semaines après une infection opportuniste dans les IRIS liés aux infections mycobactériennes et aux cryptococcoses au cours du VIH $[27,14,31]$.

\section{Paramètres orientant le diagnostic}

Certains paramètres, parfois controversés, pourraient être associés à I'IRIS lors de la survenue des symptômes et pourraient donc aider à poser le diagnostic:

- conversion de l'intradermoréaction à la tuberculine au cours des IRIS associés à la tuberculose chez les patients infectés ou non par le VIH, témoignant de la reconstitution d'une immunité spécifique fonctionnelle $[12,29]$;

- agmentation du pourcentage de CD4 ou du rapport CD4/CD8 au cours du VIH [32];

- baisse rapide de la charge virale VIH [31];

- augmentation du taux de lymphocytes totaux dans le cadre d'un IRIS associé à la tuberculose hors VIH [29];

- argmentation du taux de polynucléaires neutrophiles au-delà de $4500 / \mathrm{mm} 3$ lors de la sortie d'aplasie (PNN < 100/mm3) dans l'aspergillose pulmonaire [6];

- polymorphisme génétique en particulier de certaines cytokines (IL-6, IL-4) $[8,16,22]$.

\section{Aspects cliniques des IRIS}

De très nombreux pathogènes ont été associés à la survenue d'IRIS (Tableau II) [9]. La majorité des IRIS surviennent au cours de la tuberculose, en particulier dans le contexte d'une infection par le VIH, témoignant avant tout de la prévalence de cette infection. Les autres causes sont rares, certaines sont exceptionnelles.

\section{IRIS associés aux infections VIH}

\section{Mycobacterium tuberculosis (IRIS paradoxal rarement infectieux) ${ }^{2}$}

La tuberculose est responsable de la majorité des IRIS diagnostiqués chez les patients VIH, et la fréquence d'un IRIS au cours de cette infection varie de $8 \%$ à $43 \%$ [13]. Le délai médian de survenue des principales manifestations cliniques, adénopathies ( $63 \%$ des patients), fièvre $(39 \%)$, infiltrats pulmonaires ou épanchements pleuraux (27\%), est de 3 semaines après le début du traitement antirétroviral [13]. Chez environ $2 \%$ des patients originaires de zones de forte endémie tuberculeuse, la survenue d'un IRIS révèle une tuberculose paucisymptomatique non diagnostiquée lors de l'instauration du traitement antirétroviral [13].

\footnotetext{
${ }^{2}$ Voir la définition des trois formes d'IRIS donnée plus haut.
}

Cryptococcus neoformans (IRIS paradoxal et infectieux) La fréquence de I'IRIS paradoxal varie de $8 \%$ à $66 \%$ selon les études $[14,31]$. Les principales manifestations cliniques, méningites aseptiques (64\%), fièvre (38\%) et adénopathies nécrotiques (22\%), surviennent 3 mois (médiane) après l'introduction du traitement antirétroviral [9]. Des IRIS infectieux démasquant des infections asymptomatiques peuvent être à l'origine de méningites inhabituellement inflammatoires [9].

\section{Cytomégalovirus (IRIS paradoxal)}

Des uvéites inflammatoires sont décrites chez $38 \%$ à $63 \%$ des patients traités pour une rétinite à cytomégalovirus [9]. Elles surviennent 6 mois après l'introduction des traitements antirétroviraux (médiane).

Leucoencéphalopathie multifocale progressive (LEMP) à JC virus (IRIS paradoxal et infectieux)

La fréquence d'un IRIS paradoxal et infectieux a été estimée à $19 \%$ [33]. Des aggravations rapidement fatales ou I'apparition de LEMP ont été rapportées chez des patients auparavant asymptomatiques avec à l'IRM (imagerie à résonance magnétique) des prises de contraste et des effets de masse [34].

\section{IRIS observés hors d'un contexte d'infection VIH}

\section{IRIS et infections mycobactéries}

- M. tuberculosis (IRIS paradoxal). Le tableau clinique est comparable à celui observé lors de l'infection VIH. L'IRIS est rare en cas de tuberculose pulmonaire $(2,5 \%)$, plus fréquent en cas d'atteinte ganglionnaire (13\%-23\%) et de tuberculose disséminée (50\%$60 \%)[35]$.

\section{IRIS et cancer}

Le contexte est essentiellement celui d'hémopathies et de sorties d'aplasie après une chimiothérapie. Des syndromes de détresse respiratoire ont été décrits chez $34 \%$ des patients hospitalisés en réanimation lors d'une neutropénie profonde. L'existence d'une pneumopathie initiale, le plus souvent bactérienne, était retrouvée chez les deux tiers des patients [30].

- Aspergillus fumigatus (IRIS paradoxal). L'aggravation d'une aspergillose pulmonaire dans les 2 à 5 jours suivant la sortie d'aplasie a été observée chez $33 \%$ à $40 \%$ des patients [6].

- Candida species (IRIS de type infectieux). L'IRIS est observé chez $5 \%$ des patients ayant le plus souvent une leucémie aiguë, en sortie d'aplasie et dans le contexte d'une candidose chronique disséminée ou d'une candidose hépatosplénique [16]. 


\section{IRIS et transplantation}

- Cryptococcus neoformans (IRIS paradoxal). Une aggravation clinique est observée chez 4,8\% des patients 5 semaines et demi (médiane) après le début du traitement antifongique et surtout concomitamment à la baisse des immunosuppresseurs [5].

- CMV (IRIS paradoxal). L'apparition d'une uvéite au décours d'une rétinite à CMV est rapportée chez 0,1\% des patients transplantés après la baisse des immunosuppresseurs [24].

\section{IRIS associé à la grossesse}

- Mycobacterium tuberculosis (IRIS paradoxal). Vingtneuf cas d'IRIS ont été décrits dans le cadre d'une tuberculose survenant dans les 10 jours après la délivrance. La fréquence des atteintes extrapulmonaires (93\%) et du système nerveux central $(69 \%)$ est très élevée et à l'origine d'une mortalité et de séquelles importantes [36].

- Mycobacterium leprae (IRIS infectieux et paradoxal). Six pour cent des lèpres débutent pendant la lactation et les femmes traitées ont un risque majeur (50\%) de réaction de réversion pendant le postpartum [8].

\section{Pathologies inflammatoires et auto-immunes}

Un IRIS se rencontre dans les thyroïdites de Hashimoto; pour $45 \%$ des femmes atteintes d'une maladie de Basedow, le diagnostic a été fait en post-partum et $70 \%$ des femmes qui sont porteuses asymptomatiques d'anticorps antithyroïde pendant la grossesse développent la maladie au cours du post-partum [8].

\section{IRIS associé à l'arrêt des antagonistes du TNF $\alpha$ ou des anticorps anti-tumor necrosis factor (TNF)}

- Mycobacterium tuberculosis (IRIS paradoxal). On note une aggravation clinique après l'arrêt des anti-TNF chez 4 des 6 patients traités pour une tuberculose induite par les anti-TNF $\alpha[7]$.

\section{Traitement du syndrome de reconstitution immune}

\section{Traitement curatif}

\section{IRIS lié au VIH}

Les IRIS associés à une tuberculose et à une cryptococcose semblent évoluer favorablement sans traitement spécifique dans $30 \%$ à $45 \%$ des cas $[9,13]$ et, pour les premiers, la mortalité est inférieure à $1 \%$ [3]. Morbidité et parfois mortalité initiales sont essentiellement dues aux manifestations de I'IRIS qui touchent le sys- tème nerveux central. L'évolution à long terme des patients ayant présenté un IRIS est satisfaisante et comparable à celle des patients qui sont restés asymptomatiques après le début des ART [37].

Des recommandations thérapeutiques ont été proposées bien qu'elles n'aient pas fait l'objet d'études cliniques [38]:

- poursuite ou mise en route du traitement spécifique de l'agent infectieux à l'origine de I'IRIS;

- poursuite des ART, sauf si l'IRIS menace le pronostic vital ;

- en fonction de chaque situation clinique, on peut discuter: une abstention thérapeutique ou un traitement symptomatique (antalgique, antipyrétique); la prescription d'anti-inflammatoires non stéroïdiens, mais on dispose de très peu d'expérience clinique; la prescription d'une corticothérapie. C'est le traitement le plus utilisé pour les IRIS liés aux mycobactéries et aux cryptococcoses, mais il reste controversé pour les IRIS associés aux virus $[13,34]$. La posologie et la durée de ce traitement sont à discuter en fonction de chaque situation clinique. Une posologie de $1,5 \mathrm{mg} / \mathrm{kg} / \mathrm{j}$ de prednisone pendant 2 semaines, puis à doses décroissantes, puis $0,75 \mathrm{mg} / \mathrm{kg} / \mathrm{j}$ pendant 2 semaines avant l'arrêt a montré récemment son efficacité sur la durée des symptômes et de l'hospitalisation dans une étude randomisée contre placebo au cours des IRIS associés à la tuberculose [39]. On pèsera le risque d'infections opportunistes en particulier avec les IRIS qui n'ont pas de caractères de gravité, compte tenu de l'évolution souvent spontanément favorable.

\section{IRIS hors VIH}

L'efficacité de la corticothérapie est démontrée dans les réactions de réversion au cours de la lèpre. Elle est également efficace ( 0,5 à $1 \mathrm{mg} / \mathrm{kg} / \mathrm{j}$ pendant 3 semaines) associée au traitement antifongique dans les candidoses chroniques disséminées et permet de réduire significativement la durée des symptômes (4,5 jours contre 4 semaines) et la durée d'hospitalisation [16].

\section{Traitement préventif}

La prévention de I'IRIS infectieux chez les patients asymptomatiques repose actuellement sur la recherche systématique d'une infection asymptomatique ou pauci-symptomatique chez les patients immunodéprimés et son traitement avant la phase de reconstitution immune. II n'y a pas actuellement de recommandations pour la prévention de I'IRIS paradoxal chez des patients présentant par exemple une tuberculose disséminée. Le délai de la mise en route des ART chez les patients infectés par le VIH, qui pourrait diminuer l'incidence des IRIS, n'est pas recommandé chez les patients au stade d'infection opportuniste. En effet, le taux de mortalité double lorsque les antirétroviraux sont débutés tardivement (au-delà de 4 semaines contre moins de 2 semaines) au cours des infections opportunistes non liées aux mycobactéries [40]. Des études prospectives sont en cours dans le cas de la tuberculose.

Parmi les stratégies préventives qui devraient être prochainement évaluées, I'utilisation d'inhibiteurs du récepteur de chimiokines CCR5 (un corécepteur du VIH) dans le traitement antirétroviral pourrait, en plus de son action antirétrovirale pour les souches de VIH à tropisme R5, limiter la survenue d'IRIS par ses propriétés immunomodulatrices et antiinflammatoires, en particulier en limitant le recrutement des cellules inflammatoires au site de l'infection. 


\section{Conclusion}

L'IRIS est un événement fréquent qui survient dans des situations d'immunodépression/reconstitution très différentes. La reconnaissance d'une entité unique survenant dans des contextes multiples pourra aider à établir des liens entre des disciplines parfois éloignées, ce qui permettrait d'améliorer notre connaissance de la physiopathologie de ce syndrome. C'est aujourd'hui un prérequis pour que l'on puisse disposer de thérapies en particulier préventives, et éviter ainsi que la face sombre de I'IRIS (le syndrome inflammatoire) ne masque le bénéfice de la reconstitution immune. $\diamond$

\section{SUMMARY}

Immune reconstitution inflammatory syndrome or IRIS

The immune reconstitution inflammatory syndrome is a heterogeneous collection of pathological manifestations attributed to an excessive and deregulated immune response to various infectious and noninfectious pathogens consecutive to the modification of immune status. Mycobacteria and mycoses in the context of an HIV infection are the main causes of IRIS but this syndrome is also observed with many infectious pathogens in the context of auto-immune or inflammatory diseases. An excessive specific CD4 Thl response leading to delayed hypersensitivity with imbalanced effector and regulatory cellular immune responses is suggested. The role played by host polymorphisms remains to be investigated. The emergence of a unique syndrome gathering multiple immune-depression/reconstitution such as HIV infection and antiretroviral therapy, decrease of immune depression in transplant, recovery from neutropenia after chemotherapy for cancer, and even post-partum period, could link various specialties and help to better understand physiopathology which is crucial to elaborate preventive therapies. $\diamond$

\section{REMERCIEMENTS}

Je remercie le Pr Olivier Lortholary pour ses conseils et sa lecture critique.

\section{CONFLIT D'INTÉRÊTS}

L'auteur déclare n'avoir aucun conflit d'intérêts concernant les données publiées dans cet article.

\section{RéFÉRENCES}

1. French MA, Price P, Stone SF. Immune restoration disease after antiretroviral therapy. AIDS 2004 ; 18 : 1615-27.

2. Shelburne SA, Hamill RJ, Rodriguez-Barradas MC, et al. Immune reconstitution inflammatory syndrome: emergence of a unique syndrome during highly active antiretroviral therapy. Medicine $2002 ; 81: 213-27$

3. French MA. Immune reconstitution inflammatory syndrome: a reapparaisal. Clin Infect Dis 2009; $48: 101-7$.

4. Chen VCC, Yuen KY, Wong SSY, et al. Immunorestitution diseases in patients not infected with HIV. EurJ Clin Microbiol Infect Dis $2001 ; 20: 402-6$.

5. Singh $\mathrm{N}$, Lortholary 0 , Alexander BD, et al. An immune reconstitution syndrome-like illness associated with cryptococcus neoformans infection in organ transplant recipients. Clin Infect Dis $2005 ; 40: 1756-61$.

6. Micelli MH, Maertens J, Buvé K, et al. Immune reconstitution inflammatory syndrome in cancer patients with pulmonary aspergillosis recovering from neutropenia : proof of principle, description, and clinical and research implications. Cancer $2007 ; 110: 112-20$.
7. Garcia-Vidal C, Rodriguez Fernandez S, Martinez Lacasa J, et al. Paradoxical response to antituberculous therapy in infliximab-treated patients with disseminated tuberculosis. Clin Infect Dis $2005 ; 40$ : 756-9.

8. Singh N, Perfect JR. Immune reconstitution syndrome and exacerbation of infections after pregnancy. Clin Infect Dis 2007 ; 45 : 1192-9.

9. Breton $G$. Syndrome de reconstitution immunitaire chez les patients infectés par le VIH. La Lettre de l'Infectiologue 2006 ; 3 : 116-25.

10. Meintjes G, Lawn S, Scanio F, et al. For the international network for the study of HIV-associated IRIS. Tuberculosis-associated immune reconstitution inflammatory syndrome: case definition for use in resourcelimited settings. Lancet Infect Dis 2008 ; 8: 516-23.

11. Haddow LJ, Moosa MY, Easterbrook PJ. Validation of a published case definition for tuberculosis-associated immune reconstitution inflammatory syndrome. AIDS $2010 ; 24: 103-8$.

12. Narita M, Ashkin D, Hollender ES, Pitchenik AE. Paradoxical worsening of tuberculosis following antiretroviral therapy in patients with AIDS. Am J Respir Crit Care Med 1998; 158 : 157-61.

13. Breton $G$. Immune reconstitution inflammatory syndrome. In: Schaaf SH, Zumla A, eds. Tuberculosis: a comprehensive clinical reference. New York: Saunders-Elsevier, $2009 ; 689-700$.

14. Lortholary 0 , Fontanet $A$, Némain $\mathrm{N}$, et al. Incidence and risk factors of immune reconstitution inflammatory syndrome complicating HIVassociated cryptococcosis in France. AIDS $2005 ; 19$ : 1043-9.

15. Breton $G$, Adle-Biassette $H$, Therby $A$, et al. Immune reconstitution inflammatory syndrome in HIV-infected patients with disseminated histoplasmosis. AIDS $2006 ; 20: 119-21$.

16. Legrand F, Lecuit M Dupont B, et al. Adjuvant corticosteroid therapy for chronic disseminated candidiasis. Clin Infect Dis 2008; 46: 696-702.

17. Bourgarit A, Carcelain G, Martinez V, et al. Explosion of tuberculin-specific Thl-responses induces immune restoration syndrome in tuberculosis and HIV co-infected patients. AIDS $2006 ; 20$ : Fl-7.

18. Tan DB, Yong YK, Tan HY, et al. Immunological profiles of immune restoration disease presenting as mycobacterial lympadenitis and cryptococcal meningitis. HIV Med 2008 ; 9 : 307-16.

19. Singh $\mathrm{N}$, Lortholary 0 , Alexander BD, et al. Allograft loss in renal transplant recipients with Cryptococcus neoformans associated immune reconstitution syndrome. Transplantation $2005 ; 80: 1131-3$.

20. Seddiki N, Sasson SC, Santaner-Nanan B, et al. Proliferation of weakly suppressive regulatory CD4 T cells is associated with over-active CD4 T cell response in HIV-positive patients with mycobacterial immune restoration disease. Eur J Immunol 2009; 39 : 391-403.

21. Bourgarit A, Carcelain G, Samri A, et al. TB-associated immune restoration syndrome in HIV-1-infected patients involves tuberculinspecific CD4Thl cells and can be predicted by KIR-negative gd T cells. Montreal: $16^{\text {th }}$ Conference on retroviruses and opportunistic infections, 2009 (abstract 772).

22. Price $P$, Morahan $G$, Huang $D$, et al. Polymorphisms in cytokine genes define subpopulations of HIV-1 patients who experienced immune restoration diseases. AIDS $2002 ; 16: 2043-7$.

23. Vendrely A, Bienvenu B, Gasnault J, et al. Fulminant inflammatory leukoencephalopathy associated with HAART-induced immune restoration in AIDS-related progressive multifocal leukoencephalopathy. Acta Neuropathol $2005 ; 109: 449-55$

24. Egli A, Bergamin 0, Müllhaupt B, et al. Cytomegalovirus-associated chorioretinitis after liver transplantation: case report and review of the literature. Transpl Infect Dis 2008; $10: 27-43$.

25. Bochud Py, Hawn TR, Siddiqui MR, et al. Toll-like receptor 2 polymorphisms are associated with reversal reaction in leprosy. $J$ Infect Dis 2008 ; $197:$ 253-61.

26. Lawn SD, Myer L, Bekker LG, et al. Tuberculosis associated immune reconstitution disease: incidence, risk factors and impact in an antiretroviral treatment service in South Africa. AIDS 2007 ; 21 : 335-41.

27. Manabe $Y C$, Cambell JD, Sydnor $\varepsilon$, et al. Immune reconstitution inflammatory syndrome: risk factors and treatment implications. J Acquir Immune Defic Syndr $2007 ; 46: 456-62$.

28. Murdoch DM, Venter WDF, Feldman C, Van Rie A. Incidence and risk factors for the immune reconstitution inflammatory syndrome in HIV patients in south Africa: a prospective study. AIDS 2008; 22 : 601-10.

29. Cheng VCC, Yam WC, Woo PCY, et al. Risk factors for development of paradoxical response during antituberculosis therapy in HIV-negative patients. Eur J Clin Microbiol Infect Dis 2003 ; 22 : 597-602.

30. Azoulay $\varepsilon$, Darmon M, Delchaux C, et al. Deterioration of previous acute lung injury during neutropenia recovery. Crit Care Med 2002 ; 30 : 781-6. 


\section{RÉFÉRENCES}

31. Shelburne SA, Visnegarwala F, Darcourt, et al. Incidence and risk factors for immune reconstitution inflammatory syndrome during higly active antiretroviral therapy. AIDS $2005 ; 19: 399-406$.

32. Breton $G$, Duval $X$, Estellat $C$, et al. Determinants of immune reconstitution inflammatory syndrome in HIV type 1 -infected patients with tuberculosis after initiation of antiretroviral therapy. Clin Infect Dis 2004 ; 39 : 1709-12.

33. Cinque P, Bossolasco S, Brambilla AM, et al. The effect of HAART-induced immune reconstitution on development and outcome of progressive multifocal encephalopathy: study of 43 cases with review of the litterature. J Neurovirol $2003 ; 9: 73-80$.

34. Tan K, Roda R, Ostrow L, Mc Arthur J, Nath A. PML-IRIS in patients with HIV infection. Clinical manifestations and treatment with steroids. Neurology $2009 ; 72$ : 1458-64.

35. Cheng VCC, Ho PL, Lee RA, et al. Clinical spectrum of paradoxical deterioration during antituberculosis therapy in non-HIV-infected patients. Eur J Clin Microbiol Infect Dis $2002 ; 21: 803-9$.

36. Cheng VCC, Woo PCY, Lau SKP, et al. Peripartum tuberculosis as a form of immunorestitution disease. Eur J Clin Microb Infect Dis 2003 ; 22 : 313-7.
37. Park WB, Choe PG, Jo JH, et al. Immune reconstitution inflammatory syndrome in the first year after HAART: influence on long term outcome. AIDS $2006 ; 20: 2390-2$.

38. Kaplan JE, Benson C, Homes KK, Brooks JT, Masur T. Guidelines for prevention and treatment of opportunistics infections in HIV-infected adults and adolescents. Morb Mortal Wkly Rep (MMWR) 2009; $58: 1-198$.

39. Meintjes G, Wilkinson R, Morroni C, et al. Randomiszed placebo-controlled trial of prednisone for the TB IRIS. Montreal : $16^{\text {th }}$ Conference on retroviruses and opportunistic infections, 2009 (abstract 34).

40. Zolopa A, Anderson J, Komarow L, et al. Immediate versus deferred ART in the setting of acute AIDS-related opportunistic infection: final results of a randomized strategy trial, ACTG 5164. Boston: $15^{\text {th }}$ Conference on retroviruses and opportunistic infections, 2008 (abstract 142).

41. Catros V, Bouet F, Cabillic F, et al. Lymphocytes T gamma delta en cancérologie : des lymphocytes tueurs non conventionnels. Med Sci (Paris) $2010 ; 26$ : 185-93.

42. Leung-Theung-Long $S$, Guerder $S$. Les cellules Th17 : une nouvelle population de cellules T CD4 effectrices pro-inflammatoires. Med Sci (Paris) 2008 ; 24 : 972-6.

\section{Collection SCIENCE ET BIOMÉDECINE}

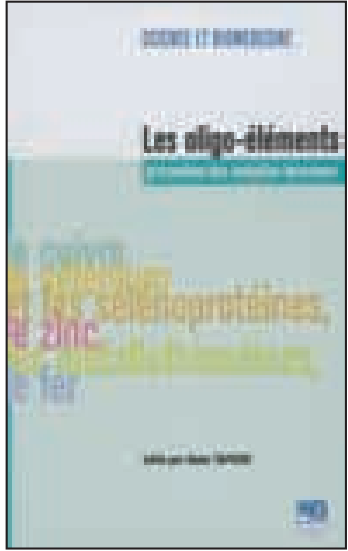

ISBN : 2-84254-107-3 64 pages

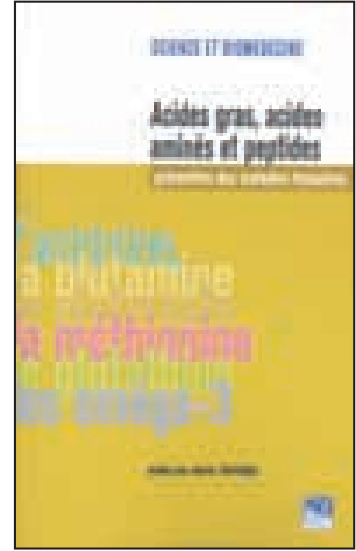

ISBN : 2-84254-108-1 80 pages

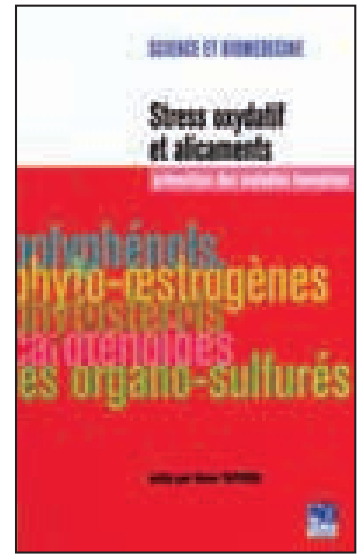

ISBN : 2-84254-111-1 86 pages

\section{Bon de commande}

À retourner à EDK, 2, rue Troyon - 92316 Sèvres Cedex

Tél. : 0155641393 - Fax : 0155641394 - E-mail : edk@edk.fr

NOM : Prénom :

Adresse :

Code postal :

Ville :

Pays :

Fonction :

Je souhaite recevoir l'ouvrage Les oligo-éléments : $10 €+3 €$ de port $=\mathbf{1 3} €$ TTC

Je souhaite recevoir l'ouvrage Acides gras, acides aminés et peptides : $12 €+3 €$ de port $=\mathbf{1 5} €$ TTC

Je souhaite recevoir l'ouvrage Stress oxydatif et alicaments : $14 €+3 €$ de port $=\mathbf{1 7} €$ TTC

en exemplaire, soit un total de

$\square$ Par chèque, à l'ordre de $\mathbf{E} \mathbf{D} \mathbf{K}$

$\square$ Par carte bancaire

$\square$ Visa $\square$ Eurocard/Mastercard

Carte $n^{\circ} \quad \begin{array}{lllllllllllllllllll}1 & 1 & 1 & 1 & 1 & 1 & 1 & 1 & 1\end{array}$

Date d'expiration : $\quad 1 \quad 1 \quad 1 \quad 1$

$\mathrm{N}^{\circ}$ de contrôle au dos de la carte :

Signature :

TIRÉS À PART

G. Breton 


\section{Hépatite B}

\section{Jean-Michel Pawlotsky Daniel Dhumeaux}

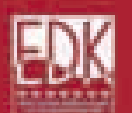

rois cent cinquante millions de porteurs chroniques dans le monde, environ un million de décès chaque année, un vaccin efficace mais décrié en France, des traitements de plus en plus performants mais inaccessibles dans les régions les plus touchées par l'endémie... Près d'un demi-siècle après la découverte du virus de l'hépatite $B$, les défis posés par l'infection restent considérables.

La publication d'un ouvrage de référence sur l'hépatite $B$, après celui sur l'hépatite $C$, s'imposait. Les meilleurs spécialistes français se sont réunis pour faire le point sur les connaissances les plus récentes, sous l'œil bienveillant de Harvey Alter, co-découvreur du virus de l'hépatite B avec Baruch Blumberg, prix Nobel de Médecine, alors que l'European Association for the Study of the Liver (EASL) publiait ses première recommandations de pratiques cliniques sur la prise en charge de l'hépatite chronique $B$ et qu'un nouveau plan de lutte contre les hépatites virales voyait le jour. Un ouvrage qui arrive bien à propos.

Jean-Michel Pawlotsky

Daniel Dhumeaux

Offre exceptionnelle réservée aux abonnés à $M / S$ jusqu'au 31 mars $2010: 54 €$

ISBN : 978-2-8425-4131-6 576 pages

À retourner à عDK, 2, rue Troyon - 92310 Sèvres

Tél. : 0155641393 - Fax : 0155641394 - ع-mail : edk@edk.fr

NOM : Prénom :

Adresse :

Code postal : Ville :

Pays :

Fonction :

Je souhaite recevoir l'ouvrage

Hépatite $B: 54 €+3 €$ de port $=\mathbf{5 7} €$ TTC

en exemplaire, soit un total de $€$

$\square$ Par chèque, à l'ordre de EDK

$\square$ Par carte bancaire : $\square$ Visa $\square$ Eurocard/Mastercard

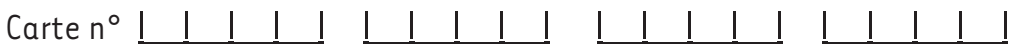
Signature :

$N^{\circ}$ de contrôle au dos de la carte:

Date d'expiration : 